\title{
Woman Giving Birth With Kyphoscoliosis Spinal Cord Injury and Placenta Previa: A Case Report
}

\author{
Tae-Hee Kim ${ }^{\mathrm{a}}$, Hae-Hyeog Lee ${ }^{\mathrm{a}, \mathrm{d}}$, Soo-Ho Chung ${ }^{\mathrm{a}}$, \\ Sun-Chul Hwang ${ }^{\mathrm{b}}$, Jun-Mo Kim ${ }^{\mathrm{c}}$
}

\begin{abstract}
Kyphoscoliosis is a spine deformity with posterior convex angulations greater than 40 degrees of a segment of vertebral column, combined with lateral and rotational deformities. Pregnancy of a patient with kyphoscoliosis is a very dangerous phenomenon. The majority of patients with spinal cord injuries involved like in this case are highly likely to have complications during pregnancy. The patient in this case suffered a spinal cord injury and kyphoscoliosis 20 years ago due to slip down. There has not been any report that a pregnant woman accompanied with kyphoscoliosis, spinal cord injury and placenta previa low-lying gave birth to a baby after 37 gestational weeks. The patient was transferred to our hospital due to cerebral hemorrhage during the confinement period, however, she gained a complete recovery under treatment and thus left our hospital. There is no exact guideline to keep pregnancy when high risk patients desire to maintain pregnancy. We report the case of a woman giving birth with kyphoscoliosis, spinal cord injury and placenta previa low-lying with a brief review of literature.
\end{abstract}

Keywords: Kyphoscoliosis; Placenta previa; Spinal cord injury

\section{Introduction}

Kyphoscoliosis is a contraindication to pregnancy, and gen-

\footnotetext{
Manuscript accepted for publication March 13, 2012

${ }^{a}$ Department of Obstetrics and Gynecology College of Medicine, Soonchunhyang University, Bucheon Hospital, Bucheon, 420-767, Republic of Korea

${ }^{b}$ Department of Neurosurgery, College of Medicine, Soonchunhyang University, Bucheon Hospital, Bucheon, 420-767, Republic of Korea ${ }^{c}$ Department of Urology, College of Medicine, Soonchunhyang University, Bucheon Hospital, Bucheon, 420-767, Republic of Korea

${ }^{\mathrm{d}}$ Corresponding author: Hae-Hyeog Lee, Department of Obstetrics and Gynecology, Soonchunhyang University Bucheon Hospital, 1174 Jung-1-dong, Wonmi-gu, Bucheon-si, Gyeonggi-do, 424-767, Republic of Korea. Email: hh122@schbc.ac.kr
}

doi:10.4021/jmc637e erally, affected women are counseled on early termination. A good outcome for the mother and baby has been reported in the majority of spinal cord injuries, but there remain some problems. Placenta previa is a risk factor for massive bleeding during a Cesarean section. No cases accompanied with these risk factors during pregnancy have been reported, yet.

\section{Case Report}

The 33-year-old woman visited to our hospital at 10 weeks gestation. According to the past history, she suffered spinal cord injury and kyphoscoliosis 20 years ago due to slip down. She showed paralysis of both the lower limbs and no significant finding in antenatal care. When she was at $37^{+5}$ weeks, she gave birth to a 3,050 gram, boy through Caesarean section with general anesthesia owing to placenta previa, and the baby had no significant finding. She discharged at 6 days after operation without significant finding. She revisited to emergency room with stupor consciousness at 14th days after delivery. She had pulmonary congestion and general spasm. Endotracheal intubation was performed.

In the brain CT scan, acute intracerebral hematoma intruding the right frontal lobe's movement area and the left frontal lobe's entire movement area was observed to be about $15 \mathrm{~mL}$ and $16 \mathrm{~mL}$, respectively, and a small amount of subarachnoid hemorrhage was observed around intracerebral hematoma (Fig. 1). Valproic acid and lasix were intravenously injected. She discharged at 44th days after delivery, and recovered completely, now.

\section{Discussion}

Spine deformity is an uncommon handicap but we must consider what the patient wants. Obstetricians usually counsel high risk patients to take early terminatioln. Kyphoscoliosis is an uncommon condition with a prevalence ranging from $0.02 \%$ to $0.7 \%$ of the US female population [1]. The primary concern is kyphoscoliosis, which may involve cardiopulmonary compromise due to mechanical restriction associated with spine deformity, and this may be further exacerbated 

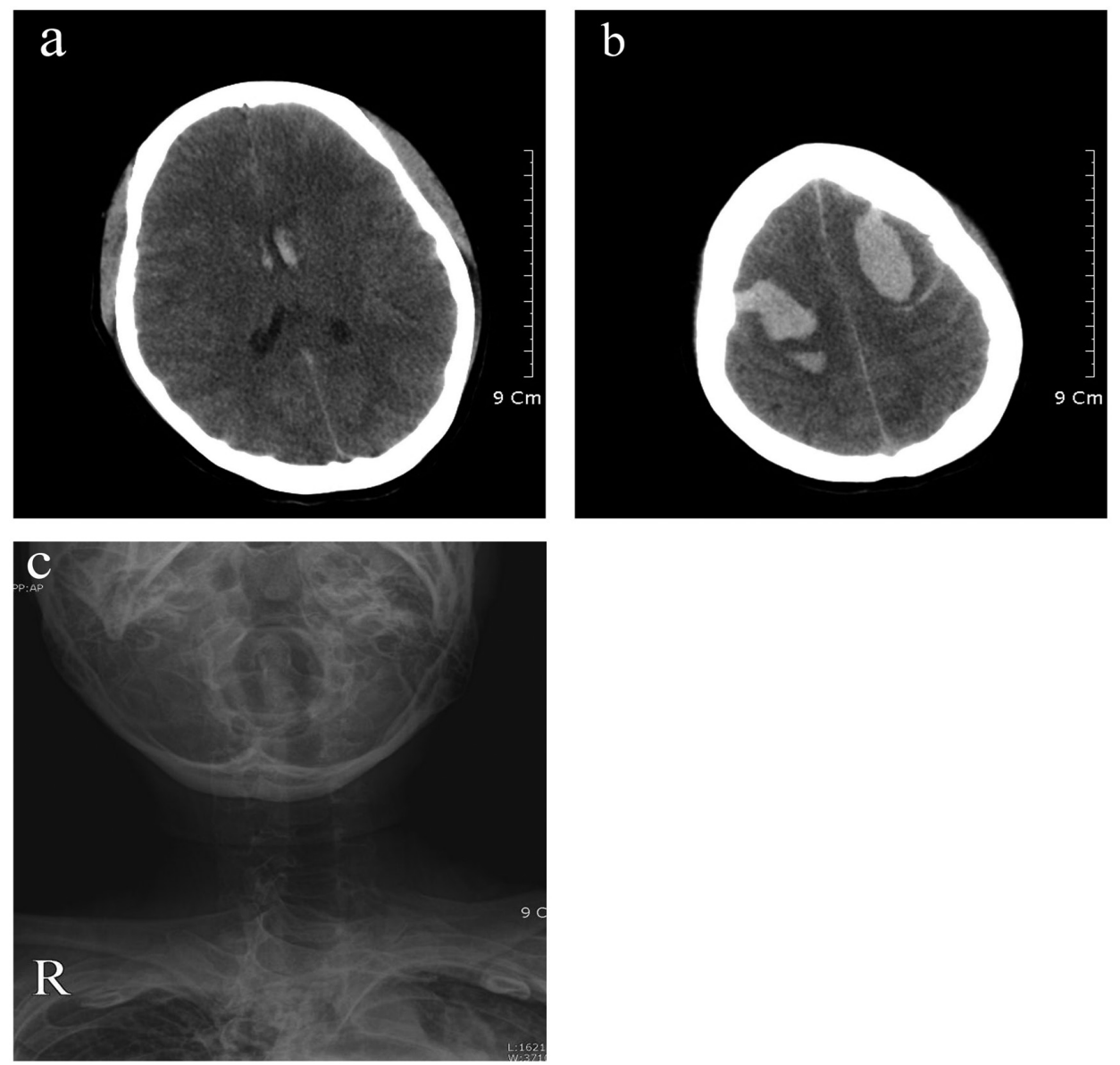

Figure 1. Brain CT scans ( $a$ and b) obtained in this patient with presence of stuporous consciousness. Axial images showing intraventricular hemorrhage (a) and intracerebral hematomas in both frontal lobes (b). The cortical sulci are effaced, which is compatible with brain edema. $X$-ray (c) shows the state where trachea is bent as much as that intubation is difficult due to serious kyphoscoliosis.

by pregnancy-related respiratory changes [2]. Therefore a pregnant woman with kyphoscoliosis has a very dangerous phenomenon. It is estimated that there are currently 20,000 women between the ages of 16 and 30 years with spinal cord injuries in the US, and each year 2,000 women of childbearing age additionally sustain spinal cord injuries in the US [3].

These diseases develop severe complications during pregnancy. The most common complication is that chronic respiratory insufficiencies due to chest wall deformation, such as kyphoscoliosis, are likely to increase during pregnancy and cause respiratory failure [4]. Spinal cord injury patients suffer from urinary tract infections, anemia, venous thrombosis, pulmonary embolism, preterm labor, and decubitus ulcers during pregnancy [5]. It is important that a team approach is needed according to gestations of pregnancy. Chest physicians and obstetricians are aware of the risks of respiratory failure associated with pregnancy in kyphoscoliosis patients with apparently stable but serious [6]. We divide management into three stages. In the first stage, we need teams composed of obstetricians, radiologists involved in fetal sonography and chest physicians until $34^{+6}$ gestational week. It is too difficult for obstetricians to detect fetal abnormality, because patients with kyphoscoliosis cannot move and change easily to see the fetus during sonography. Therefore radiologists involved in fetal sonography must take care of the fetus during the patient's pregnancy. After 34 weeks, we must consider delivery time. We could no delivery at 34 weeks, because the patient wants to keep the fetus until 37 weeks. This is our first mistake. She was stable but we have to consider to delivery at 34 weeks. In the second stage of delivery, we need teams composed of anesthesiologists, chest physicians and a skillful surgery team. The patient underwent a Cesarean section with intubation under general anesthesia. There have been some reports of benefit of non-invasive ventilator support [6]. The skillful surgery team had to reduce the operation time. In the third stage of postpartum period, we need teams composed of anesthesiologists, chest physicians, cardiologists and infectious 
disease specialists. Regular monitoring with capillary blood gas analysis and ventilatory support in the intensive care unit should be considered. In the postpartum period, the paraplegic patients are at an increased risk of urinary tract infection, thrombophlebitis, and pulmonary embolism [7]. At 7 days after operation, she was stable and complained of dyspnea as usual. Then she was discharged. Pregnancy and the puerperium are associated with an increased risk of stroke, and stroke is considered as an important cause of maternal morbidity and mortality during this time. Pregnancy and delivery can lead to substantial alterations in systemic arterial and venous hemodynamics and may predispose to cerebrovascular disorders [8]. The mother of this case may have experienced hemorrhagic stroke by increasing venous pressure within the cranial cavity when cardiac load pressure of the cardio pulmonary increased due to blood transfusion after hemorrhage from placenta previa along with the finding of anemia of the complication of a spinal cord injury during pregnancy under the circumstances of pulmonary function impairment and cardiopulmonary compromise, which are the complications of kyphoscoliosis. The author recommends different team approaches and management for a high risk patient into three stages. We do not give up the fetus but recommend termination. This case is reported as a mother having kyphoscoliosis and a spinal cord injury, who received operation for placenta previa posing risk of hemorrhage, where any one of them is a serious risk factor during pregnancy, did not show any significant finding, and then was recovered 2 weeks after operation for cerebral hemorrhage.

\section{Consent}

Written informed consent was obtained from our patient for publication of this case report and accompanying images. A copy of the written consent is available for review by the Editor-in-Chief of this journal.

\section{Competing Interests}

The authors declare that they have no competing interests.

\section{Authors' Contributions}

All authors conceived the study concept and design, collected clinical data, reviewed the literature on the topic, and drafted the manuscript. All authors have read and approved the final manuscript.

\section{References}

1. Smith MW, Marcus PS, Wurtz LD. Orthopedic issues in pregnancy. Obstetrical \& gynecological survey. 2008;63(2):103-111.

2. To WW, Wong MW. Kyphoscoliosis complicating pregnancy. International journal of gynaecology and obstetrics: the official organ of the International Federation of Gynaecology and Obstetrics. 1996;55(2):123-128.

3. Ghidini A, Healey A, Andreani M, Simonson MR. Pregnancy and women with spinal cord injuries. Acta obstetricia et gynecologica Scandinavica. 2008;87(10):1006-1010.

4. Gamzu R, Shenhav M, Fainaru O, Almog B, Kupferminc $\mathrm{M}$, Lessing JB. Impact of pregnancy on respiratory capacity in women with muscular dystrophy and kyphoscoliosis. A case report. The Journal of reproductive medicine. 2002;47(1):53-56.

5. Baker ER, Cardenas DD. Pregnancy in spinal cord injured women. Archives of physical medicine and rehabilitation. 1996;77(5):501-507.

6. Reddy R, Evans E, Khoo O, Allen MB. Pregnancy in kyphoscoliosis: benefit of non-invasive ventilatory support. Journal of obstetrics and gynaecology : the journal of the Institute of Obstetrics and Gynaecology. 2005;25(3):267-268.

7. Crosby E, St-Jean B, Reid D, Elliott RD. Obstetrical anaesthesia and analgesia in chronic spinal cord-injured women. Canadian journal of anaesthesia $=$ Journal canadien d'anesthesie. 1992;39(5 Pt 1):487-494.

8. Bateman BT, Schumacher HC, Bushnell CD, Pile-Spellman J, Simpson LL, Sacco RL, Berman MF. Intracerebral hemorrhage in pregnancy: frequency, risk factors, and outcome. Neurology. 2006;67(3):424-429. 